Bull. Austral. Math. Soc.

VOL. 59 (1999) [247-255]

\title{
TORAL AUTOMORPHISMS AND ANTIAUTOMORPHISMS OF ROTATION ALGEBRAS
}

\author{
Hu Yaohua and P.J. STACEY
}

\begin{abstract}
If $U, V$ are the generators of a rational or irrational rotation $C^{*}$-algebra then an automorphism $\phi$ of the algebra is determined by $\phi(U)=\lambda U^{a} V^{c}$ and $\phi(V)=\mu U^{b} V^{d}$ where $\lambda, \mu$ are complex numbers of modulus 1 and $a, b, c, d$ are integers with $a d-b c=$ 1. If $a d-b c=-1$, then these formulae determine an antiautomorphsm of the algebra. The classification of such automorphisms and antiautomorphisms up to conjugacy by arbitrary automorphisms is studied and an almost complete classification is obtained.
\end{abstract}

\section{INTRODUCTION}

Let $A_{\theta}$ be the universal $C^{*}$-algebra generated by a pair $U, V$ of unitaries satisfying $V U=\rho U V$ where $\rho=e^{2 \pi i \theta}$ and $0 \leqslant \theta<1$. Brenken [2] and Watatani [8] introduced an $\mathrm{SL}(2, \mathbb{Z})$ action on $A_{\theta}$, which can be described in the following way. If $A \in \operatorname{SL}(2, \mathbb{Z})$ and $\lambda, \mu \in S^{1}$ then an automorphism $\phi_{A, \lambda, \mu}$ of $A_{\theta}$ is determined, using the universal property, by $\phi_{A, \lambda, \mu}(U)=\lambda U^{a} V^{c}$ and $\phi_{A, \lambda, \mu}(V)=\mu U^{b} V^{d}$, where $A=\left(\begin{array}{ll}a & b \\ c & d\end{array}\right)$. Following Watatani, such automorphisms of $A_{\theta}$ will be called toral. The particular choice $\lambda=e^{\pi i a c}$ and $\mu=e^{\pi i b d}$ gives an $\mathrm{SL}(2, \mathbb{Z})$ action by toral automorphisms.

A series of papers by Farsi and Watling, including [3] and [4], obtained properties of the fixed point algebras and cross product algebras associated with toral automorphisms. The purpose of the present paper is to include a study of antiautomorphisms and to study the conjugacy problem for both toral automorphisms and antiautomorphisms.

\section{CONJUGACY OF TORAL AUTOMORPHISMS}

The description of toral automorphisms up to conjugacy was mostly completed in the paper [4]. The following propositions make those results explicit as well as completing the description. Under the isomorphism of $K_{1}\left(A_{\theta}\right)$ with $\mathbb{Z}^{2}$ given by $\left[U^{n} V^{m}\right] \mapsto(n, m)$ the map $\left(\phi_{A, \lambda, \mu}\right)_{*}$ corresponds to multiplication by $A$ and hence a necessary condition for $\phi_{A, \lambda, \mu}$ and $\phi_{A^{\prime}, \lambda^{\prime}, \mu^{\prime}}$ to be conjugate automorphisms is that $A$ and $A^{\prime}$ are conjugate in $\mathrm{SL}(2, \mathbb{Z})$. The following results specify the extra conditions needed to ensure conjugacy of the automorphisms.

Received 22nd July, 1998

Copyright Clearance Centre, Inc. Serial-fee code: 0004-9729/99 \$A2.00+0.00. 
Proposition 1. (Farsi and Watling). If $\operatorname{trace}(A) \neq 2$ then $\phi_{A, \lambda, \mu}$ is conjugate to $\phi_{A, 1,1}$. Furthermore $\phi_{A, 1,1}$ is conjugate to $\phi_{B, 1,1}$ if and only if $A$ is conjugate to $B$ in $S L(2, \mathbb{Z})$.

Proof: This is established in the course of the proofs of $[4$, Proposition 3 and Lemma 4].

If $\operatorname{trace}(A)=2$ then it is well-known that $A$ is conjugate in $\operatorname{SL}(2, \mathbb{Z})$ to $M=\left(\begin{array}{cc}1 & m \\ 0 & 1\end{array}\right)$ for some $m \in \mathbb{Z}$ and that $m$ is uniquely determined. The following explicit description of the conjugacy when $m \neq 0$ is needed for Proposition 2. Let $A=\left(\begin{array}{cc}1-a & b \\ c & 1+a\end{array}\right)$ with $a^{2}=-b c$. If $c=0$ then $A$ is already of the required form (with $a=0$ ), if $b=0$ then $\left(\begin{array}{cc}0 & 1 \\ -1 & 0\end{array}\right)\left(\begin{array}{cc}1-a & 0 \\ c & 1+a\end{array}\right)\left(\begin{array}{cc}0 & -1 \\ 1 & 0\end{array}\right)=\left(\begin{array}{cc}1+a & -c \\ 0 & 1-a\end{array}\right)$ is of the required form (with $a=0$ ) and otherwise each of $a, b, c$ are non-zero. In the latter case let $|m|=(b, c)$ with $b m>0$. From the condition $a^{2}=-b c$ it follows that $b=r^{2} m$ and $c=-t^{2} m$ for some $r ; t \in \mathbb{Z}$ with $(r, t)=1$. Furthermore the signs of $r, t$ can be chosen so that $a=m r t$. If $u, v$ are chosen to satisfy $r u-t v=1$ then $A=\left(\begin{array}{cc}r & v \\ t & u\end{array}\right)\left(\begin{array}{cc}1 & m \\ 0 & 1\end{array}\right)\left(\begin{array}{cc}u & -v \\ -t & r\end{array}\right)$. This last equation and hence the equations $a=m r t, b=r^{2} m$ and $c=-t^{2} m$ hold even when $b=0$ or $c=0$.

Proposition 2. (i) (Farsi and Watling). If $A=\left(\begin{array}{cc}1-a & b \\ c & 1+a\end{array}\right), a=$ mrt, $b=r^{2} m$ and $c=-t^{2} m$ with $(r, t)=1$ and $m \neq 0$ then $\phi_{A, \lambda, \mu}$ is conjugate to $\phi_{M, \gamma, 1}$ where $M=\left(\begin{array}{cc}1 & m \\ 0 & 1\end{array}\right)$ and $\gamma=\lambda^{r} \mu^{t} \rho^{r\left[-c-a c-a-a^{2}\right] / 2}$.

(ii) $\phi_{M, \gamma, 1}$ is conjugate to $\phi_{M, \gamma^{\prime}, 1}$ if and only if $\gamma=\gamma^{\prime}$ or $\gamma=\gamma^{\prime-1}$.

Proof: (i) This is shown in the proof of [4, Lemma 16].

(ii) Suppose that $\phi_{M, \gamma, 1} \beta=\beta \phi_{M, \gamma^{\prime}, 1}$ for some automorphism $\beta$ of $A_{\theta}$. Then $\phi_{M, \gamma, 1} \beta(U)=\gamma^{\prime} \beta(U)$. Following the proof of [3, Theorem 1], let $c_{p, q}$ be the Fourier coefficient of $\beta(U)$ corresponding to $U^{p} V^{q}$, defined to be the usual Fourier coefficient of $E\left(\beta(U) V^{* q}\right)$ corresponding to $U^{p}$, where $E$ is the canonical conditional expectation from $A_{\theta}$ onto $C^{*}(U)$. Then $\sum \gamma^{\prime} c_{p, q} U^{p} V^{q}=\sum \gamma^{p} c_{p, q} U^{p}\left(U^{m} V\right)^{q}=\sum \gamma^{p} c_{p, q} \rho^{s} U^{p+q m} V^{q}$ for some $s$, so $\left|c_{p, q}\right|=\left|c_{p+q m, q}\right|$ for each $p, q$ and thus, since $\left|c_{p, q}\right| \rightarrow 0$ as $p \rightarrow \infty, c_{p, q}=0$ unless $q=0$. It follows that $\beta(U)$ has Fourier series $\sum c_{p} U^{p}$ and that $c_{p} \gamma^{\prime}=c_{p} \gamma^{p}$ for each $p$. If $\gamma$ has infinite order then $c_{p}$ is non-zero for only one value of $p$ and thus $\beta(U)=c_{p} U^{p}$. If $\gamma$ has order $k$ then $\beta(U)=U^{p} f\left(U^{k}\right)$ for some $f: S^{1} \rightarrow S^{1}$ and some $p$ such that $\gamma^{\prime}=\gamma^{p}$. If $\beta_{*}: K_{1}\left(A_{\theta}\right) \rightarrow K_{1}\left(A_{\theta}\right)$ corresponds to $\beta$ then, when $K_{1}\left(A_{\theta}\right)$ is identified with $\mathbb{Z}^{2}$ in the usual way, it follows that $\beta_{*}(1,0)=(p+k \ell, 0)$ for some $\ell \in \mathbb{Z}$ (or $\beta_{*}(1,0)=(p, 0)$ when $\gamma$ has infinite order). On the other hand, from $\phi_{M, \gamma, 1} \beta=\beta \phi_{M, \gamma^{\prime}, 1}$ it follows that $M B=B M$ 
where $\beta_{*}$ has matrix $B$. However if $\left(\begin{array}{ll}a & b \\ c & d\end{array}\right)\left(\begin{array}{cc}1 & m \\ 0 & 1\end{array}\right)=\left(\begin{array}{ll}1 & m \\ 0 & 1\end{array}\right)\left(\begin{array}{ll}a & b \\ c & d\end{array}\right)$ then $c=0$ and $a=d$ and so, from $a d-b c=1, B= \pm\left(\begin{array}{ll}1 & b \\ 0 & 1\end{array}\right)$ for some $b \in \mathbb{Z}$. Hence $\beta_{*}(1,0)= \pm(1,0)$ and so $(p+k \ell, 0)= \pm(1,0)$. If $k \neq 1$ (that is, $\gamma \neq 1$ ) then $\ell=0$ and $p= \pm 1$ whereas if $k=1$ then $\gamma^{\prime}=\gamma=1$. Thus in all cases $\gamma=\gamma^{\prime \pm 1}$. Conversely, if $\gamma^{\prime}=\gamma^{-1}$ and $\beta$ is defined by $\beta(U)=\rho^{m} U^{*}$ and $\beta(V)=V^{*}$ then $\phi_{M, \gamma, 1} \beta=\beta \phi_{M, \gamma^{\prime}, 1}$, as required.

The final case to be considered is when $A$ is conjugate to, and hence equal to, $I_{2}$. The behaviour of $\phi_{A, \lambda, \mu}$ depends on the nature of the subgroup $G$ of $S^{1}$ generated by $\lambda, \mu$.

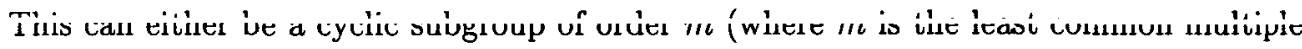
of the orders of $\lambda, \mu$ ), an infinite cyclic group (when $\lambda$ or $\mu$ has infinite order and $\lambda^{k}=\mu^{\ell}$ for some $k, \ell$ not both zero), a group $\mathbb{Z}_{m} \times \mathbb{Z}$ where $1<m$ (when $\lambda^{k} \mu^{-\ell}$ is of finite order for some $k, \ell$, not both zero, but either $\lambda$ or $\mu$ has infinite order) or $\mathbb{Z} \times \mathbb{Z}$ (when $\lambda^{k} \mu^{-\ell}$ is of infinite order unless $k=\ell=0$ ).

Propos ITION 3. (i) (Farsi and Watling). If $\lambda, \mu$ generate a cyclic subgroup of order $m$ in $S^{1}$ then $\phi_{I, \lambda, \mu}$ is conjugate to $\phi_{I, \omega, 1}$ where $\omega=e^{2 \pi i / m}$.

(ii) If $\lambda, \mu$ generate an infinite cyclic subgroup in $S^{1}$ then $\phi_{I, \lambda, \mu}$ is conjugate to $\phi_{I, 1, \gamma}$ for some $\gamma$ of infinite order in $S^{1}$. Furthermore $\phi_{I, 1, \gamma}$ is conjugate to $\phi_{I, 1, \gamma^{\prime}}$ if and only if $\gamma=\gamma^{\prime}$ or $\gamma=\gamma^{\prime-1}$.

(iii) If $\lambda, \mu$ generate a subgroup of $S^{1}$ isomorphic to $\mathbb{Z}_{m} \times \mathbb{Z}$ then $\phi_{I, \lambda, \mu}$ is conjugate to $\phi_{I, \omega^{n}, \gamma}$ for some $n$ with $1 \leqslant n<m$ and $(n, m)=1$ and some $\gamma$ of infinite order, where $\omega=e^{2 \pi i / m}$. Furthermore $\phi_{I, \omega^{n}, \gamma}$ is conjugate to $\phi_{I, \omega^{n^{\prime}}, \gamma^{\prime}}$ if and only if either $\omega^{n}=\omega^{n^{\prime}}$ and $\gamma=\omega^{s} \gamma^{\prime}$ for some $s \in \mathbb{Z}$ or $\omega^{n}=w^{-n^{\prime}}$ and $\gamma=\omega^{s} \gamma^{\prime-1}$ for some $s \in \mathbb{Z}$.

(iv) If $\lambda, \mu$ generate a subgroup of $S^{\mathbf{1}}$ isomorphic to $\mathbb{Z} \times \mathbb{Z}$ then the toral automorphisms conjugate to $\phi_{I, \lambda, \mu}$ are those of the form $\phi_{I, \lambda^{a} \mu^{b}, \lambda^{r} \mu^{\circ}}$ where $s a-r b=1$.

Proof: (i) From the proof of $\left[4\right.$, Theorem 20] there exists $A=\left(\begin{array}{ll}a & b \\ c & d\end{array}\right) \in \operatorname{SL}(2, \mathbb{Z})$ such that $\lambda^{a} \mu^{c}=\omega$ and $\lambda^{b} \mu^{d}=1$. Then $\phi_{I, \lambda, \mu} \phi_{A, 1,1}=\phi_{A, 1,1} \phi_{I, \omega, 1}$, as required.

(ii), (iii). Let $\lambda^{k} \mu^{-\ell}$ be a generator of $\left\{1, \ldots, \omega^{m-1}\right\}$ (where $m=1$ in case (ii)), let $k^{\prime}=k /(k, \ell)$ and let $\ell^{\prime}=\ell /(k, \ell)$. Then in case (ii) $\lambda^{k^{\prime}} \mu^{-\ell^{\prime}}=1$ and in case (iii) $\lambda^{k^{\prime}} \mu^{-\ell^{\prime}}=\omega^{n}$ for some $1 \leqslant n<m$ with $(n, m)=1$. There exist $b, d \in \mathbb{Z}$ with $d k^{\prime}+b \ell^{\prime}=1$ and then $A=\left(\begin{array}{cc}k^{\prime} & b \\ -\ell^{\prime} & d\end{array}\right) \in \mathrm{SL}(2, \mathbb{Z})$ and $\phi_{I, \lambda, \mu} \phi_{A, 1,1}=\phi_{A, 1,1} \phi_{I, \omega^{n}, \lambda^{b} \mu^{d}}$ (with $n=0$ in case (ii)).

Suppose that there exists an automorphism $\beta$ with $\beta \phi_{I, \omega^{n^{\prime}}, \gamma^{\prime}}=\phi_{I, \omega^{n}, \gamma} \beta$ (with $n=$ $n^{\prime}=0$ in case (ii)). If the Fourier coefficient of $\beta(U)$ corresponding to $U^{r} V^{s}$ is $c_{r, s}$ then $\omega^{n^{\prime}} c_{r, s}=\omega^{n r} \gamma^{s} c_{\tau, s}$ and so, since $\gamma$ is of infinite order, $c_{r, s}=0$ unless $s=0$. Thus 
$\beta(U) \in C^{*}(U)$. Similarly, if the Fourier coefficient of $\beta(V)$ corresponding to $U^{s} V^{t}$ is $d_{s, t}$ then $\gamma^{\prime} d_{s, t}=\omega^{s n} \gamma^{t} d_{s, t}$ and, since $\gamma$ is of infinite order, there exists a unique $t$ such that $d_{s, t} \neq 0$. Thus $\beta(V)=W V^{t}$ for some $W \in C^{*}(U)$. The matrix corresponding to $\beta_{*}: K_{1}\left(A_{\theta}\right) \rightarrow K_{1}\left(A_{\theta}\right)$ is therefore of the form $\left(\begin{array}{ll}* & * \\ 0 & t\end{array}\right)$ and, since $\beta_{*}$ is invertible, it follows that $t= \pm 1$. Hence $\gamma=\gamma^{\prime}$ or $\gamma=\gamma^{\prime-1}$ in case (ii) and $\gamma=\omega^{s} \gamma^{\prime}$ for some $s \in \mathbb{Z}$ or $\gamma=\omega^{s} \gamma^{\prime-1}$ for some $s \in \mathbb{Z}$ in case (iii).

By reversing the roles of $n, \gamma$ and $n^{\prime}, \gamma^{\prime}$, it follows that not only $\beta(U) \in C^{*}(U)$ but also $\beta^{-1}(U) \in C^{*}(U)$. Hence the automorphism $\beta$ restricts to an automorphism of $C^{*}(U)$ implementing a conjugacy between the restrictions of $\phi_{I, \omega^{n^{\prime}}, \gamma^{\prime}}$ and $\phi_{I, \omega^{n}, \gamma}$. However unless $\omega^{n}=\omega^{ \pm n^{\prime}}$, the associated rational rotation algebras are non-isomorphic. The case $\omega^{n}=w^{n^{\prime}}$ corresponds to $\beta_{*}[U]=[U]$ and hence to $t=1$, whereas $\omega^{n}=\omega^{-n^{\prime}}$ corresponds to $\beta_{*}[U]=-[U]$ and hence to $t=-1$. Thus either $\omega^{n}=\omega^{n^{\prime}}$ and $\gamma=\omega^{s} \gamma^{\prime}$ for some $s \in \mathbb{Z}$ or $\omega^{n}=\omega^{-n^{\prime}}$ and $\gamma=\omega^{s} \gamma^{\prime-1}$ for some $s \in \mathbb{Z}$.

Conversely, in case (iii) let $\beta(U)=U$ and $\beta(V)=U^{s^{\prime}} V$ where $n s^{\prime} \equiv s(\bmod m)$, which exists because $(n, m)=1$. Then $\phi_{I, \omega^{n}, \gamma} \beta=\beta \phi_{I, \omega^{n}, \omega^{s} \gamma}$. Similarly let $\beta(U)=U^{*}$ and $\beta(V)=V^{*}$ in case (ii) to obtain $\phi_{I, 1, \gamma} \beta=\beta \phi_{I, 1, \gamma^{-1}}$ or $\beta(U)=U^{*}$ and $\beta(V)=U^{s^{\prime}} V^{*}$ in case (iii) to obtain $\phi_{I, \omega^{n}, \gamma} \beta=\beta \phi_{I, \omega^{-n}, \omega^{s} \gamma^{-1}}$.

(iv) Let $\phi_{I, \lambda, \mu} \beta=\beta \phi_{I, \lambda^{\prime}, \mu^{\prime}}$. Then $\phi_{I, \lambda, \mu} \beta(U)=\lambda^{\prime} \beta(U)$ and $\phi_{I, \lambda, \mu} \beta(V)=\mu^{\prime} \beta(V)$ so if the Fourier coefficient of $\beta(U)$ corresponding to $U^{a} V^{b}$ is $c_{a, b}$ and that of $\beta(V)$ corresponding to $U^{r} V^{s}$ is $d_{r, s}$, then $\lambda^{\prime} c_{a, b}=\lambda^{a} \mu^{b} c_{a, b}$ and $\mu^{\prime} d_{r, s}=\lambda^{r} \mu^{s} d_{r, s}$. Since no two distinct pairs $\lambda^{a} \mu^{b}$ are equal it follows that $\beta(U)=c_{a, b} U^{a} V^{b}$ for some $a, b$ and similarly $\beta(V)=d_{r, s} U^{r} V^{s}$. Hence $\lambda^{\prime}=\lambda^{a} \mu^{b}, \mu^{\prime}=\lambda^{r} \mu^{s}$ and, from the invertibility of $\beta_{*}$ on $K_{1}\left(A_{\theta}\right), s a-r b=1$. Conversely, if $a, b, r, s$ satisfy the condition $s a-r b=1$ then $\phi_{I, \lambda, \mu} \beta=\beta \phi_{I, \lambda^{a} \mu^{b}, \lambda^{r} \mu^{s}}$ when $\beta(U)=U^{a} V^{b}$ and $\beta(V)=U^{r} V^{s}$.

\section{ANTIAUTOMORPHISMS}

In order to establish the existence of an antiautomorphism of $A_{\theta}$, observe that the opposite algebra $A_{\theta}^{o p}$ is generated by the elements $U, V$ but subject to the relation $V U=\bar{\rho} U V$ rather than $V U=\rho U V$. Hence $A_{\theta}^{o p}$ is isomorphic to $A_{1-\theta}$ and, by the universal property, there exists an isomorphism $\alpha$ from $A_{\theta}$ onto $A_{\theta}^{o p}$ with $\alpha(V)=U$ and $\alpha(U)=V$. Composing this with the identity antiautomorphism from $A_{\theta}^{o p}$ to $A_{\theta}$ gives an antiautomorphism of $A_{\theta}$, denoted by $\phi_{B, 1,1}$ where $B=\left(\begin{array}{ll}0 & 1 \\ 1 & 0\end{array}\right) \in G L(2, \mathbb{Z})$. If $A=\left(\begin{array}{ll}a & b \\ c & d\end{array}\right) \in G L(2, \mathbb{Z})$ with $a d-b c=-1$ then the composite $\phi_{A B, \mu, \lambda} \phi_{B, 1,1}$ is an antiautomorphism $\phi_{A, \lambda, \mu}$ with $\phi_{A, \lambda, \mu}(U)=\lambda U^{a} V^{c}$ and $\phi_{A, \lambda, \mu}(V)=\mu U^{b} V^{d}$. 
Proposition 4. (i) If $A, A^{\prime} \in G L(2, \mathbb{Z})$ with $\operatorname{det}(A)=1$ then

$$
\phi_{A, \lambda, \mu} \phi_{A^{\prime}, \lambda^{\prime}, \mu^{\prime}}=\phi_{A A^{\prime}, \gamma, \delta}
$$

where

$\gamma=\lambda^{\prime} \lambda^{a^{\prime}} \mu^{c^{\prime}} \rho^{\left(b d c^{\prime}\left(c^{\prime}-1\right)\right) / 2+\left(a c a^{\prime}\left(a^{\prime}-1\right)\right) / 2+a^{\prime} b c c^{\prime}}$ and $\delta=\mu^{\prime} \lambda^{b^{\prime}} \mu^{d^{\prime}} \rho^{\left(b d d^{\prime}\left(d^{\prime}-1\right)\right) / 2+\left(a b^{\prime} c\left(b^{\prime}-1\right)\right) / 2+b b^{\prime} c d^{\prime \prime}}$

(ii) If $A, A^{\prime} \in G L(2, \mathbb{Z})$ with $\operatorname{det}(A)=-1$ then

$$
\phi_{A, \lambda, \mu} \phi_{A^{\prime}, \lambda^{\prime}, \mu^{\prime}}=\phi_{A A^{\prime}, \gamma, \delta}
$$

where

$\gamma=\lambda^{\prime} \lambda^{a^{\prime}} \mu^{c^{\prime}} \rho^{\left(b d c^{\prime}\left(c^{\prime}-1\right)\right) / 2+\left(a a^{\prime} c\left(a^{\prime}-1\right)\right) / 2+a a^{\prime} c^{\prime} d}$ and $\delta=\mu^{\prime} \lambda^{b^{\prime}} \mu^{d^{\prime}} \rho^{\left(b d d^{\prime}\left(d^{\prime}-1\right)\right) / 2+\left(a b^{\prime} c\left(b^{\prime}-1\right)\right) / 2+a b^{\prime} d d^{\prime}}$

(iii) The map from $A=\left(\begin{array}{ll}a & b \\ c & d\end{array}\right)$ to $\psi_{A}=\phi_{A, \exp (\pi i a c), \exp (\pi i b d)}$ is a $G L(2, \mathbb{Z})$ action by toral automorphisms and antiautomorphisms.

(iv) When $G L(2, \mathbb{Z})$ acts on $T^{2}$ by $(\lambda, \mu) \theta_{A}=\left(\lambda^{a} \mu^{c}, \lambda^{b} \mu^{d}\right)$, where $A=\left(\begin{array}{ll}a & b \\ c & d\end{array}\right)$, then $\phi_{I, \lambda, \mu} \psi_{A}=\psi_{A} \phi_{I,(\lambda, \mu) \theta_{A}}$ and hence $(\lambda, \mu, A) \mapsto \psi_{A} \phi_{I, \lambda, \mu}$ is an action of the semidirect product $T^{2} \rtimes G L(2, \mathbb{Z})$.

Proof: These are all straightforward calculations.

A major component of the classification of the toral antiautomorphisms up to conjugacy is the classification up to conjugacy of elements in $G L(2, \mathbb{Z})$ with negative determinant. If two such elements $A, B$ satisfy $A=Q B Q^{-1}$ with $Q \in G L(2, \mathbb{Z})$ then, replacing $Q$ by $Q B$ if necessary, they are conjugate by some $Q \in \mathrm{SL}(2, \mathbb{Z})$.

The following result shows that, as for automorphisms when $\operatorname{trace}(A) \neq 2$, the conjugacy problem for toral antiautomorphisms (via automorphisms) when $\operatorname{trace}(A) \neq 0$ reduces to the conjugacy problem in $G L(2, \mathbb{Z})$.

Proposition 5. If $\operatorname{trace}(A) \neq 0$ and $\operatorname{det}(A)=-1$ then $\phi_{A, \lambda, \mu}$ is conjugate to $\phi_{A, 1,1}$. Furthermore $\phi_{A, 1,1}$ is conjugate to $\phi_{B, 1,1}$ if and only if $A$ is conjugate to $B$ in $G L(2, \mathbb{Z})$.

Proof: Let $A=\left(\begin{array}{ll}a & b \\ c & d\end{array}\right)$, let $\gamma=\lambda^{(1-d) /(a+d)} \mu^{c /(a+d)}$ and let $\delta=\lambda^{b /(a+d)} \mu^{(1-a) /(a+d)}$. Then $\phi_{A, \lambda, \mu} \phi_{I, \gamma, \delta}=\phi_{I, \gamma, \delta} \phi_{A, 11}$. If $A=Q B Q^{-1}$ with $Q \in S L(2, \mathbb{Z})$ then $\phi_{Q, 1,1} \phi_{B, 1,1} \phi_{Q, 1,1}^{-1}=$ $\phi_{A, \lambda, \mu}$ for some $\lambda, \mu \in S^{1}$ and hence, by the first part, is conjugate to $\phi_{A, 1,1}$.

If $A \in G L(2, \mathbb{Z})$ with $\operatorname{det}(A)=-1$ and $\operatorname{trace}(A)=0$ then $A^{2}=I$. Hence, from [5, Lemma 5.5, p.166], $A$ is conjugate to either $M=\left(\begin{array}{cc}1 & 0 \\ 0 & -1\end{array}\right)$ or $N=\left(\begin{array}{ll}0 & 1 \\ 1 & 0\end{array}\right)$ (which are not conjugate to one another). 
PRoposition 6. If $\operatorname{trace}(A)=0$ then either $\phi_{A, \lambda, \mu}$ is conjugate to $\phi_{M, \gamma, 1}$ for some $\gamma \in S^{1}$ or to $\phi_{N, \gamma, 1}$ for some $\gamma \in S^{1}$.

ProOF: Let $Q \in \mathrm{SL}(2, \mathbb{Z})$ with $Q A Q^{-1}=M$ (or $Q A Q^{-1}=N$ ). Then $\phi_{Q, 1,1} \phi_{A, \lambda, \mu} \phi_{Q, 1,1}^{-1}=\phi_{M, \lambda^{\prime}, \mu^{\prime}}$ for some $\lambda^{\prime}, \mu^{\prime} \in S^{1}$ (or $\phi_{N, \lambda^{\prime}, \mu^{\prime}}$ for some $\lambda^{\prime}, \mu^{\prime} \in S^{1}$ ). However $\phi_{I, \mu, 1} \phi_{N, \lambda \mu, 1}=\phi_{N, \lambda, \mu} \phi_{I, \mu, 1}$ and, if $\delta^{2}=\mu$, then $\phi_{I, 1, \delta} \phi_{M, \lambda, \mu}=\phi_{M, \lambda, 1} \phi_{I, 1, \delta}$.

PROPOSITION 7. $\phi_{N, \gamma, 1}$ is conjugate to $\phi_{N, \gamma^{\prime}, 1}$ if and only if $\gamma=\gamma^{\prime}$ or $\gamma=\gamma^{\prime-1}$.

PRoOF: Let $\beta \phi_{N, \gamma, 1}=\phi_{N, \gamma^{\prime}, 1} \beta$. Then $\beta \phi_{I, \gamma, \gamma}=\beta \phi_{N, \gamma, 1}^{2}=\phi_{N, \gamma^{\prime}, 1}^{2} \beta=\phi_{I, \gamma^{\prime}, \gamma^{\prime}} \beta$. Thus when $\gamma$ has infinite order then, by Proposition 3(ii), either $\gamma=\gamma^{\prime}$ or $\gamma=\gamma^{\prime}-1$. When $\gamma$ has finite order $m$ then, by Proposition $3(\mathrm{i}), \gamma=\gamma^{n}$ for some $1 \leqslant n<m$ with $(n, m)=1$. Then $\phi_{I, \gamma^{\prime}, \gamma^{\prime}}\left(U^{* n} \beta(U)\right)=\gamma^{\prime-n} U^{* n} \phi_{I, \gamma^{\prime}, \gamma^{\prime}} \beta(U)=\gamma^{-1} U^{* n} \beta \phi_{I, \gamma, \gamma}(U)=U^{* n} \beta(U)$ and similarly $\phi_{I, \gamma^{\prime}, \gamma^{\prime}}\left(V^{* n} \beta(V)\right)=V^{* n} \beta(V)$, from which it follows that $U^{* n} \beta(U) \in F i x\left(\phi_{I, \gamma^{\prime}, \gamma^{\prime}}\right)=$ $C^{*}\left(U^{m}, U V^{*}\right)$ and $V^{* n} \beta(V) \in C^{*}\left(U^{m}, U V^{*}\right)$. Hence the induced map $\beta_{*}$ on $K_{1}\left(A_{\theta}\right) \cong \mathbb{Z}^{2}$ satisfies $\beta_{*}(1,0)=(n+\ell m+k,-k)$ for some $\ell, k \in \mathbb{Z}$ and $\beta_{*}(0,1)=\left(k^{\prime}+\ell^{\prime} m, n-k^{\prime}\right)$ for some $\ell^{\prime}, k^{\prime} \in \mathbb{Z}$ that is,

$$
\beta_{*}=\left(\begin{array}{cc}
n+\ell m+k & k^{\prime}+\ell^{\prime} m \\
-k & n-k^{\prime}
\end{array}\right)
$$

However $\beta_{*} N=N \beta_{*}$, where $N=\left(\begin{array}{ll}0 & 1 \\ 1 & 0\end{array}\right)$, from which it follows that $\beta_{*} \in\left\{ \pm\left(\begin{array}{ll}1 & 0 \\ 0 & 1\end{array}\right), \pm\left(\begin{array}{ll}0 & 1 \\ 1 & 0\end{array}\right)\right\}$. If $\beta_{*}= \pm I$, it follows that $k=0$ and $n+\ell m= \pm 1$ and hence $n=1$ or $m-1$, as required. Similarly, if $\beta_{*}= \pm N$ then $k^{\prime}+\ell^{\prime} m= \pm 1$ and $n=k^{\prime}$, so $n=1$ or $m-1$, as required. Conversely, $\phi_{-I, 1,1} \phi_{N, \gamma, 1}=\phi_{N, \gamma^{-1}, 1} \phi_{-I, 1,1}$ for each $\gamma \in S^{1}$.

PROPOSITION 8. (i) If $\phi_{M, \gamma, 1}$ is conjugate to $\phi_{M, \gamma^{\prime}, 1}$ then $\gamma^{2}=\gamma^{\prime 2}$ or $\gamma^{2}=\gamma^{\prime}-2$ (ii) If $\gamma=\gamma^{\prime}$ or $\gamma=\gamma^{\prime-1}$ then $\phi_{M, \gamma, 1}$ is conjugate to $\phi_{M, \gamma^{\prime}, 1}$.

PRoof: (i) Let $\beta \phi_{M, \gamma, 1}=\phi_{M, \gamma^{\prime}, 1} \beta$. Then $\beta \phi_{I, \gamma^{2}, 1}=\beta \phi_{M, \gamma, 1}^{2}=\phi_{M, \gamma^{\prime}, 1}^{2} \beta=\phi_{I, \gamma^{\prime 2}, 1} \beta$. Thus, when $\gamma$ has infinite order then, by Proposition 3(ii), either $\gamma^{2}=\gamma^{\prime 2}$ or $\gamma^{2}=\gamma^{\prime-2}$. When $\gamma^{2}$ has finite order $m>1$ then, by Proposition 3(i), $\gamma^{2}=\gamma^{\prime 2 n}$ for some $1 \leqslant n<$ $m$ with $(n, m)=1$. Then $\phi_{I, \gamma^{\prime 2}, 1}\left(U^{* n} \beta(U)\right)=U^{* n} \beta(U)$ and $\phi_{I, \gamma^{\prime 2}, 1} \beta(V)=\beta(V)$ so $U^{* n} \beta(U) \in C^{*}\left(U^{m}, V\right)$ and $\beta(V) \in C^{*}\left(U^{m}, V\right)$. Hence $\beta_{*}: K_{1}\left(A_{\theta}\right) \rightarrow K_{1}\left(A_{\theta}\right)$ equals $\left(\begin{array}{cc}n+k m & m k^{\prime} \\ \ell & \ell^{\prime}\end{array}\right)$ for some $k, \ell, k^{\prime}, \ell^{\prime} \in \mathbb{Z}$. However, the condition $\beta_{*} M=M \beta_{*}$ implies $\beta_{*} \in\left\{ \pm\left(\begin{array}{ll}1 & 0 \\ 0 & 1\end{array}\right), \pm\left(\begin{array}{cc}1 & 0 \\ 0 & -1\end{array}\right)\right\}$ and hence $n=1$ or $m-1$. Finally, when $\gamma^{2}=1$ then $\gamma^{\prime 2}=1$, so the condition also holds in this case.

(ii) This is immediate since $\phi_{-I, 1,1} \phi_{M, \gamma, 1}=\phi_{M, \gamma^{-1,1}} \phi_{-I, 1,1}$. 
The sole remaining question is therefore whether or not $\phi_{M, \gamma, 1}$ is conjugate to $\phi_{M,-\gamma, 1}$.

Proposition 9. (i) Let $\gamma$ be of infinite order in $S^{1}$. If $\phi_{M, \gamma, 1}$ is conjugate to $\phi_{M,-\gamma, 1}$ then $\phi_{M, \lambda, 1}$ is conjugate to $\phi_{M,-\lambda, 1}$ for all $\lambda \in S^{1}$.

(ii) Let $\gamma$ be either of odd order $k$ or of order $2 k$ where $k$ is odd. If $\phi_{M, \gamma, 1}$ is conjugate to $\phi_{M,-\gamma, 1}$ then $\phi_{M, 1,1}$ is conjugate to $\phi_{M,-1,1}$.

ProOF: (i) Suppose that $\phi_{M, \gamma, 1} \beta=\beta \phi_{M,-\gamma, 1}$, where $\beta$ is an automorphism. Then $\phi_{M, \gamma, 1} \beta(U)=-\gamma \beta(U)$ and $\phi_{I, \gamma^{2}, 1} \beta(U)=\gamma^{2} \beta(U)$ and hence, if $c_{n, m}$ is the Fourier coefficient of $\beta(U)$ corresponding to $U^{n} V^{m}$ then $\gamma^{2} c_{n, m}=\gamma^{2 n} c_{n, m}$, so $c_{n, m}=0$ unless $n=1$, and $-\gamma c_{n, m} U^{n} V^{m}=\gamma^{n} \rho^{m n} c_{n,-m} V^{m} U^{n}$, so $c_{1, m}=-\rho^{m} c_{1,-m}$. It follows that $\beta(U)=U \sum c_{m} V^{m}$ where $\sum c_{m} V^{m}$ is a unitary for which $c_{m}=-\rho^{m} c_{-m}$ for each $m \in \mathbb{Z}$.

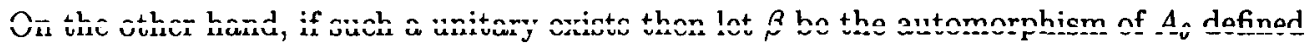
by $\beta(U)=U \sum c_{m} V^{m}$ and $\beta(V)=V$. Then, for any $\lambda \in S^{1}, \phi_{M, \lambda, 1} \beta=\beta \phi_{M,-\lambda, 1}$, as required.

(ii) If $k$ is odd and $\gamma$ is of order $k$, then $\phi_{M, \gamma, 1}^{k}=\phi_{M, 1,1}$ whereas $\phi_{M,-\gamma, 1}^{k}=\phi_{M,-1,1}$. If $\gamma$ is of order $2 k$ where $k$ is odd then $\gamma^{k}=-1$ and hence $\phi_{M, \gamma, 1}^{k}=\phi_{M,-1,1}$ and $\phi_{M,-\gamma, 1}^{k}=$ $\phi_{M, 1,1}$.

Propos It ION 10. (i) The real algebra $\left\{a \in A_{\theta}: \phi_{M, 1,1}(a)=a^{*}\right\}$ is isomorphic to the crossed product $C\left(S^{1}, \mathbb{R}\right) \times_{\alpha_{\theta}} \mathbb{Z}$ where $\alpha_{\theta}$ is the irrational rotation on $C\left(S^{1}, \mathbb{R}\right)$, defined by $\left(\alpha_{\theta} f\right)(t)=f(\rho t)$.

(ii) The real algebra $\left\{a \in A_{\theta}: \phi_{M,-1,1}(a)=a^{*}\right\}$ is isomorphic to the crossed product $\left\{f \in C\left(S^{1}\right): f(t)=\overline{f(-t)}\right.$ for all $\left.t \in S^{1}\right\} \times_{\alpha_{\theta}} \mathbb{Z}$.

Proof: When $A_{\theta}$ is viewed as $C\left(S^{1}\right) \times_{\alpha_{\theta}} \mathbb{Z}$ via the identification of $C^{*}(U)$ with $C\left(S^{1}\right)$, then $\phi_{M, 1,1}$ restricts to the identity on $C\left(S^{1}\right)$ whereas $\phi_{M,-1,1}$ restricts to the automorphism $\phi$ given by $(\phi f)(t)=f(-t)$. The results are therefore immediate.

PROPOS ITION 11. Let $R_{-1}$ be the real algebra associated with $\phi_{M,-1,1}$ and let $R_{1}$ be the real algebra associated with $\phi_{M, 1,1}$. Then $K_{0}\left(R_{1}\right)$ is isomorphic to $\mathbb{Z}^{2} \times \mathbb{Z}_{2}$ whereas $K_{0}\left(R_{-1}\right)$ is isomorphic to $\mathbb{Z}^{2}$.

Proof: The $K$-theory of $R_{1}=C\left(S^{1}, \mathbb{R}\right) \times_{\alpha} \mathbb{Z}$ is calculated in [7, Corollary 1.5.7]. The $K$-theory of $A \times_{\alpha} \mathbb{Z}$, where $A=\left\{f \in C\left(S^{1}\right): f(t)=\overline{f(-t)}\right.$ for all $\left.t \in S^{1}\right\}$, can be calculated using the Pimsner-Voiculescu sequence for real $C^{*}$-algebras, described in $[7,1.4 .2]$. Note firstly that the involution $t \mapsto-t$ on $S^{1}$ gives rise to the real $K$-theory $K R\left(S^{2,0}\right)$ of Atiyah [1] and that, by [1, (3.6)], there is an exact sequence

$$
\ldots \rightarrow K R^{2-q}(p t) \rightarrow K R^{-q}(p t) \rightarrow K R^{-q}\left(S^{2,0}\right) \rightarrow K R^{3-q}(p t) \rightarrow \ldots
$$

Using the values for $K R^{-n}(p t)$ from [6, Chapter III, Theorem 5.19], yields the values $K_{4}(A)=K_{0}(A)=\mathbb{Z}, K_{5}(A)=K_{1}(A)=\mathbb{Z}_{2}, K_{6}(A)=K_{2}(A)=0$ and $K_{7}(A)=$ $K_{3}(A)=\mathbb{Z}$. From the real Pimsner-Voiculescu sequence,

$$
\ldots \rightarrow K_{0}(A) \stackrel{\ominus}{\rightarrow} K_{0}(A) \rightarrow K_{0}\left(A \times_{\alpha} \mathbb{Z}\right) \rightarrow K_{7}(A) \stackrel{\theta}{\rightarrow} K_{7}(A) \rightarrow \ldots
$$


where $\theta=\alpha_{*}-i d$. Since $\alpha_{*}[1]=[1]$ it is immediate that, on $K_{0}(A), \alpha_{*}=i d$. From the identification of $K_{1}$ classes with winding numbers, it follows that, on $K_{7}\left(C\left(S^{1}\right)\right)=$ $K_{1}\left(C\left(S^{1}\right)\right), \alpha_{*}=i d$. However the action of $\alpha_{*}$ on $K_{7}(A)$ commutes with the complexification map $c: K_{7}(A) \rightarrow K_{7}\left(C\left(S^{1}\right)\right)$, from which it follows, using $K_{7}(A)=\mathbb{Z}$ and $K_{7}\left(C\left(S^{1}\right)\right)=\mathbb{Z}$, that $\alpha_{*}=i d$ on $K_{7}(A)$. Hence,

$$
0 \rightarrow K_{0}(A) \rightarrow K_{0}\left(A \times_{\alpha} \mathbb{Z}\right) \rightarrow K_{7}(A) \rightarrow 0
$$

from which it follows that $K_{0}\left(R_{-1}\right)=\mathbb{Z}^{2}$, as claimed.

PROPOSITION 12. $\phi_{M, \gamma, 1}$ is not conjugate to $\phi_{M,-\gamma, 1}$ when $\gamma$ is either of infinite order, odd order or of order $2 k$ where $k$ is odd.

Proof: It follows from Proposition 9 that if $\phi_{M, \gamma, 1}$ is conjugate to $\phi_{M,-\gamma, 1}$ then $\phi_{M, 1,1}$ is conjugate to $\phi_{M,-1,1}$. Hence, in the notation of Proposition $11, R_{1}$ is isomorphic to $R_{-1}$, which contradicts that Proposition.

The final remaining problem is to determine whether or not $\phi_{M, \gamma, 1}$ is conjugate to $\phi_{M,-\gamma, 1}$ when the order of $\gamma$ is a multiple of 4 . The following proposition describes how the problem can be resolved in certain cases when $\theta$ is rational. Recall that, when $\theta=p / q$ with $(p, q)=1$ and $0 \leqslant p<q$, then the algebra $A_{\theta}$ can be identified with the $C^{*}$-subalgebra of $C_{b}\left(\mathbb{R}^{2}, M_{q}\right)$ generated by $U, V$ where $U(\lambda, \mu)=e^{2 \pi i \lambda / q} U_{0}$ and $V(\lambda, \mu)=$ $e^{2 \pi \mu / q} V_{0}$ with

$$
U_{0}=\left(\begin{array}{cccc}
1 & & & \\
& \rho & & \\
& & \ddots & \\
& & & \rho^{q-1}
\end{array}\right) \text { and } V_{0}=\left(\begin{array}{cc}
O & I_{q-1} \\
1 & 0
\end{array}\right) .
$$

If $\gamma=e^{2 \pi i m / n}$ with $(m, n)=1$, then the antiautomorphism $\phi_{M, \gamma, 1}$ is given, in this description, by $\left(\phi_{M, \gamma, 1} f\right)(\lambda, \mu)=f(\lambda+(m q / n),-\mu)^{t r}$, where $t r$ denotes the transpose. To see this, note that this formula defines an antiautomorphism of $C_{b}\left(\mathbb{R}^{2}, M_{q}\right)$ mapping $U$ to $\gamma U$ and $V$ to $V^{*}$.

The algebra $A_{\theta}$ can be explicitly described as $\left\{f \in C\left(\mathbb{R}^{2}, M_{q}\right): f(\lambda+m, \mu+n)=\right.$ $\left.W_{1}^{n} W_{2}^{m} f(\lambda, \mu) W_{2}^{* m} W_{1}^{* n}\right\}$, where $W_{1}=U_{0}^{p^{\prime}}$ and $W_{2}=V_{0}^{p^{\prime \prime}}$ with $p p^{\prime} \equiv-1(\bmod q)$ and $p p^{\prime \prime} \equiv 1(\bmod q)$, from which it follows that, for each $f \in A_{\theta},(\lambda, \mu) \mapsto \operatorname{det} f(\lambda, \mu)$ gives a well-defined function on $T^{2}$. When restricted to unitary elements it gives a mapping from $T^{2}$ to $S^{1}$. The winding numbers $w_{1}(f), w_{2}(f)$ of the associated maps $\lambda \mapsto \operatorname{det} f(\lambda, 0)$ and $\mu \mapsto \operatorname{det} f(0, \mu)$ are homotopy invariants and hence (recalling that $K_{1}\left(A_{\theta}\right)$ is isomorphic to $\mathbb{Z}^{2}$ with generators $[U]$ and $\left.[V]\right)$ the $K_{1}$ element $[f]$ corresponding to the unitary element $f$ of $A_{\theta}$ is $\left(w_{1}(f), w_{2}(f)\right)$.

PROPOSITION 13. Let $\theta=p / q$ where $(p, q)=1$ and let $\gamma$ have order $n$ where $(q, n)=1$. Then, for each unitary $f$ in $A_{\theta}$ with $\phi_{M, \gamma, 1}(f)=\gamma^{r} f$, the $K_{1}$ class $[f]$ belongs to $(r+n \mathbb{Z}) \times \mathbb{Z}$. 
PROOF: Let $\gamma=e^{2 \pi i m / n}$ where $(m, n)=1$. As described above, $f(\lambda+(m q / n),-\mu)^{t r}$ $=\gamma^{r} f(\lambda, \mu)$ for each $\lambda, \mu \in \mathbb{R}$ and hence $\operatorname{det} f(\lambda+(m q / n), 0)=\gamma^{r q} \operatorname{det} f(\lambda, 0)$. Let $m^{\prime} m \equiv 1(\bmod n)$, so that $\operatorname{det} f(\lambda+(q / n), 0)=\gamma^{r q m^{\prime}} \operatorname{det} f(\lambda, 0)=e^{2 \pi i r q / n} \operatorname{det} f(\lambda, 0)$ and then $\operatorname{det} f(\lambda+(1 / n), 0)=e^{2 \pi i r / n} \operatorname{det} f(\lambda, 0)$ using $(q, n)=1$. The winding number of $\left\{\operatorname{det} f(\lambda, 0): \lambda_{0} \leqslant \lambda \leqslant \lambda_{0}+1 / n\right\}$ followed by $\left\{e^{-2 \pi i r t / n}: 0 \leqslant t \leqslant 1\right\}$ is a continuous function of $\lambda_{0}$ and hence constant, say $N$. Then the winding number of $\lambda \mapsto \operatorname{det} f(\lambda, 0)$ is $r+n N$, so $[f] \in(r+n \mathbb{Z}) \times \mathbb{Z}$.

Corollary . $\phi_{M, \gamma, 1}$ is not conjugate to $\phi_{M,-\gamma, 1}$ when $\gamma$ is of order $4 k$ and $\theta=p / q$ with $(p, q)=1$ and $(q, 4 k)=1$, unless $k=1$.

Proof: If $\phi_{M, \gamma, 1} \beta=\beta \phi_{M,-\gamma, 1}$ then $\phi_{M, \gamma, 1} \beta(U)=-\gamma \beta(U)=\gamma^{2 k+1} \beta(U)$ and hence,

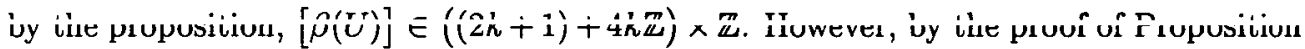
$8(\mathrm{i}),[\beta(U)]= \pm(1,0)$. Hence $k=1$ (and $\beta(U)=(-1,0))$.

The situation $k=1$ occurring in the last corollary always gives $\phi_{M, \gamma, 1}$ conjugate to $\phi_{M,-\gamma, 1}$, for then $\gamma= \pm i$ and so $-\gamma=\gamma^{-1}$. We conjecture that this is the only situation in which $\phi_{M, \gamma, 1}$ is conjugate to $\phi_{M,-\gamma, 1}$.

\section{REFERENCES}

[1] M.F. Atiyah, ' $K$-theory and reality', Quart. J. Math. Oxford Ser. 217 (1966), 367-86.

[2] B. Brenken, 'Representations and automorphisms of the irrational rotation algebra', $\mathrm{Pa}$ cific J. Math. 111 (1984), 257-282.

[3] C. Farsi and N. Watling, 'Trivial fixed point subalgebras of the rotation algebra', Math. Scand. 72 (1993), 298-302.

[4] C. Farsi and N. Watling, ' $C$-algebras of dynamical systems on the non-commutative torus', Math. Scand. 75 (1994), 101-110.

[5] D.E. Flath, Introduction to number theary (J. Wiley \& Sons, New York, 1989).

[6] M. Karoubi, K-theory: an introduction (Springer-Verlag, Berlin, Heidelberg, New York, 1978).

[7] H. Schröder, $K$-theory for real $C^{*}$-algebras and applications, Pitman Research Notes on Mathematics 290 (Pitman, New York, 1993).

[8] Y. Watatani, 'Toral automorphisms on the irrational rotation algebra', Math. Japon. 26 (1981), 479-484.

School of Mathematics

La Trobe University

Bundoora Vic 3083

Australia

e-mail: matyh@lure.latrobe.edu.au

P.Stacey@latrobe.edu.au 